\title{
Estimating Multiple Consumer Segment Ideal Points from Context-Dependent Survey Data
}

\author{
WAYNE S. DESARBO \\ A. SELIN ATALAY \\ DAVID LEBARON \\ SIMON J. BLANCHARD*
}

\begin{abstract}
Previous research in marketing and consumer research has shown that consumers/ households often possess multiple ideal points in a given product/service category. In such cases, traditional segmentation and positioning models that estimate a single ideal point per individual/segment may render an inaccurate portrayal of the true underlying utility functions of such consumers/segments and the resulting market structure. We propose a new clusterwise multiple-ideal-point spatial methodology that estimates multiple ideal points at the market segment level while simultaneously determining the market segments' composition of consumers, as well as the corresponding joint space.
\end{abstract}

$\mathrm{T}$ he consumer behavior literature supports the notion that consumers do not typically have stable utility functions. Instead, they construct their final utility assessments spontaneously as they face specific decision problems. Moreover, these on-the-spot judgments are shaped by the needs and goals of the individual, the aspects of the consumptive situation, the context in which the choice alternatives are being evaluated, how the choice problem is stated, and how the choice alternatives are framed (Belk 1974, 1975, 1979; Bettman, Luce, and Payne 1998). Thus, consumer decisions are a function of both the context in which the decisions are made and the individual making the decision (Carlson and Bond 2006; Simon 1955, 1990; Tversky and Kahneman

*Wayne S. DeSarbo is the Smeal Distinguished Research Professor of Marketing in the Marketing Department in the Smeal College of Business, 433 Business Building, Pennsylvania State University, University Park, PA 16802 (wsd6@psu.edu). A. Selin Atalay is an assistant professor of marketing at the School of Business Administration at Bilkent University, 06800 Bilkent Ankara, Turkey (atalay@bilkent.edu.tr). David LeBaron is a doctoral candidate in the Marketing Department in the Smeal College of Business, 432 Business Building, Pennsylvania State University, University Park, PA 16802 (dn1117@psu.edu). Simon J. Blanchard is a doctoral candidate in the Marketing Department in the Smeal College of Business, 447B Business Building, Pennsylvania State University, University Park, PA 16802 (sxb975@psu.edu). The authors wish to thank the editor, associate editor, and three reviewers for their insightful comments that improved this manuscript. The authors also wish to acknowledge Joon Park and Crystal Scott for their efforts in the data collection. Requests for information or reprints can be sent to Wayne S. DeSarbo at wsd6@psu.edu.

John Deighton served as editor and Brian Ratchford served as associate editor for this article.

Electronically published March 28, 2008
1991). Hence, consumers can employ a different decision strategy each time they make a decision. As a result, consumers may have different preference judgments for the same brand across different situations or contexts (Bettman et al. 1998). As consumer utility functions and decisionmaking strategies change over contexts, consumers can have more than one ideal product/point in a given product category. It is therefore essential to understand this aspect of consumer preferences because it can affect how products are promoted to consumers, how new products are designed, and how products are priced and bundled.

Previous consumer research has shown that consumers and households often possess multiple ideal points in a given product/service category. When they do, measures of market-level preferences need to account not only for heterogeneity across decision makers but also for the heterogeneity within a decision maker across time or context. We propose a new clusterwise multiple-ideal-point spatial methodology that estimates multiple ideal points at the market segment level while simultaneously determining the market segments' composition of consumers, as well as the corresponding joint space. Thus, the research question that this manuscript attempts to explicitly address is how to represent in a single conditional utility function the manner in which preferences for alternatives vary across choice contexts within segments of demand. As noted by Lee, Sudhir, and Steckel (2002), traditional multidimensional unfolding (MDU) spatial representations are extremely limited for such applications in marketing and consumer research in that such methods typically assume that each consumer/segment has a single dominating ideal product. An 
ideal product refers here to a bundle of features (product attributes) that a consumer would most like to see in a product. Indeed, this assumption of a single well-defined dominance/utility function is clearly violated in those consumer research applications where the household or family is the unit of analysis, as households usually consist of multiple consumers who possess their own individual distinct perceptions, needs, and preferences (Gupta and Steckel 1993; Kahn, Morrison, and Wright 1986; Lee et al. 2002). Consider the category of breakfast cereals. Kellogg's, for example, manufactures All-Bran, Apple Jacks, Cocoa Krispies, Complete, Kellogg's Corn Flakes, Corn Pops, Cracklin' Oat Bran, Crispix Crunch, Eggo, Froot Loops, Frosted Mini-Wheats, Honey Smacks, Kellogg's Frosted Flakes, Kellogg's Raisin Bran, Kellogg's Smorz, Low Fat Granola, Mini-Swirlz, Mueslix, Product 19, Rice Krispies, Shrek, Smart Start, and Special K cereals. It is very probable that Kellogg's notices that brand and type of cereal purchased varies across age-based market segments. Thus, within a given household, for breakfast, children may consume one formulation (e.g., presweetened cereals such as Fruit Loops), parents another (e.g., a healthy natural cereal such as Low Fat Granola), and grandparents still a different set of brands (e.g., a low sodium, high fiber cereal such as All-Bran). Thus, within such household units, multiple ideal products may exist. And, in the example above, it is to Kellogg's advantage to understand such multiple preference structures within different market segments, especially concerning multiple brand purchases and consumption.

An alternative justification for such multiple ideal points deals with the effects of situational or context factors generated in different usage or purchase situations/occasions (Belk 1974, 1975, 1979; Dubow 1992; Laurent 1978). Such context or situational effects on consumer utility functions have been well documented in the consumer research literature with actual taxonomies of usage/situation occasions having been developed (Belk 1979; Srivastava, Shocker, and Day 1978; Stefflre 1979). The need for situation-specific ideal products has been mentioned in DeSarbo and Carroll (1981a, 1981b, 1985), Hagerty (1980), and Holbrook (1984). For example, let us consider brands of soft drinks. PepsiCo sells over 50 brands of different types of beverages spanning soft drinks/soda, teas and coffees, fruit juices, waters, sports drinks, and so on. It is very likely that PepsiCo understands that consumer segments vary their preferences and consumption depending upon usage occasion (e.g., one segment may prefer Gatorade after a strenuous workout, Naked Juice as a mixer with some alcoholic drinks, Mug for use with ice cream in a float, Diet Pepsi as a complement to a meal, etc., although a different market segment may have a different pattern of usage across these same situations). Thus, multiple situation-specific ideal points may exist for different consumers/segments.

Finally, as mentioned in Lee et al. (2002), another potential source of multiple ideal points is variety seeking, which implies that consumers/segments may have different ideal products at different times or usage occasions. Re- peated consumption of the same product/service may produce saturation or boredom effects for the consumer. For example, most consumers do not frequent the same restaurant for every meal that is purchased outside the home because of variety-seeking reasons, even if the restaurant in question is their favorite. Rather, there exist multiple desirable restaurants that may specialize in a particular cuisine, and many consumers vary their consumption among these favorites according to their particular tastes on that occasion. This is perhaps one major reason why Restaurants International manages Taco Bell, KFC, Pizza Hut, Long John Silver's, and A\&W restaurants, where there are these five brands with five distinct menu positions: Mexican, chicken, pizza, fish/seafood, and American. Their goal is to capture as much share of the fast food wallet as possible, recognizing consumer segments' heterogeneous tastes and need for variety seeking.

Lee et al. (2002) proposed a stochastic multiple-idealpoint model to estimate multiple ideal products from an analysis of choice data (switching data) over time periods. This important contribution deviates substantially from previous MDU approaches. The basic premise behind their proposed model is that households possess a set of ideal products, each of which represents a distinct utility. At any given purchase occasion, one of these ideal points is activated with some probability, which results in a choice selection. Their procedure is an individual-household-level model used to estimate spatially the number of ideal points per household, their locations, brand coordinate locations, and the probabilities with which these ideal points are activated. A stagewise estimation procedure is devised using individual household brand switching matrices in a maximum likelihood estimation framework. More specifically, their computationally intensive method cycles over each and every household using a consistent Akaike's information criterion (CAIC) heuristic (see Wedel and Kamakura 2000) to determine the number of ideal points per household. After such estimation, a separate average linkage clustering procedure is then used to group these individual household ideal points to form a market-level joint-space map where market segments of multiple ideal products are jointly represented (as ideal points) with brand locations for a representation of market structure. Limitations exist with this approach relating to problems of incidental parameters, enormous amounts of required computation, insufficient justification for the parametric assumptions invoked in the approach, the disjointed relationship between the maximum-likelihood-based estimation procedure and average linkage clustering, the requirement of somewhat long purchase histories and the corresponding assumption of stationarity, and so on.

We feel that the concept of multiple ideal points has been a major contribution to the field of consumer research and MDU. We propose an alternative approach to the estimation of these multiple ideal points for simultaneous positioning and market segmentation with the use of survey data (it would be very difficult to measure such context-dependent preferences described earlier via observable panel/scanner 
choice data). Although consumer behavior research examines consumers at the individual level, marketing decisions that affect each of these consumers are typically made at the segment level. In particular, we devise a metric threeway clusterwise MDU spatial model devised to simultaneously estimate a joint-space map of brands and segmentlevel ideal points that vary by time, purchase or usage occasion, context, goal, situation, and so on. Like traditional deterministic MDU methods, the proposed methodology is deterministic and does not require parametric assumptions (unlike latent class MDU methods). In addition, the procedure can be estimated with consumer survey preference data, and long histories of data are not required. Segmentlevel ideal points and brand positions are simultaneously derived, as well as the classification of consumers/households into these derived market segments - all of which performed in a parsimonious manner to optimize a common loss function. The proposed methodology allows for either partitions or overlapping market segments as well and involves the estimation of much smaller numbers of parameters than previous MDU approaches, with much less computational effort.

The next section provides a technical description of the model, estimation framework, and the alternating leastsquares algorithm for parameter estimation (details of the algorithm are in app. A, available in the online version of $J C R)$. Next, we illustrate the use of the proposed methodology via a student study that collected perceived effectiveness judgments for some 11 major brands of over-thecounter (OTC) analgesics over a number of common maladies (e.g., contexts or consumptive situations). The results of the proposed methodology are described with associated inferences made. A comparison is made with the most flexible (in terms of providing for segment and dimensional heterogeneity in which the number and type of segments, dimensions, or both can vary by context) twostage estimation approach in terms of overall fit values. Finally, we provide a discussion of future research needs in this area and generalize the procedure to the individual level, as well as to accommodating segment and dimensional heterogeneity.

\section{PROPOSED THREE-WAY CLUSTERWISE MDU PROCEDURE}

Given that the application we will be discussing concerns the analysis of consumer perceived effectiveness judgments of various OTC analgesic brands over various consumptive contexts/situations/goals (maladies), we will use this scenario to technically define the parameters of the proposed methodology below. Note, however, that this proposed methodology can be used for the analysis of any type of two- or three-way metric dominance type data as typically collected in consumer research.

\section{Model}

Let $i=1, \ldots, N$ consumers; $j=1, \ldots, J$ brands; $t=$ $1, \ldots, T$ contexts/situations; $s=1, \ldots, S$ market segments (unknown); $r=1, \ldots, R$ dimensions (unknown); and $\Delta_{i j t}=$ the reverse scaled preference judgment (dispreference) for brand $j$ given by consumer $i$ in context/situation $t$. The full model can be written as

$$
\Delta_{i j t}=a_{t} \sum_{s=1}^{S} P_{i s} \sum_{r=1}^{R}\left(X_{j r}-Y_{s r t}\right)^{2}+b_{t}+\varepsilon_{i j t},
$$

where $X_{j r}=$ the $r$ th coordinate for brand $j, \underline{X}=\left(\left(X_{j r}\right)\right)$, $Y_{s r t}=$ the $r$ th coordinate of the ideal point for market segment $s$ in context/situation $t, Y=\left(\left(\left(Y_{s r t}\right)\right)\right), a_{t}=$ a multiplicative coefficient for context/situation $t, b_{t}=$ an additive constant for context/situation $t$, and $P_{i s}=1$ if consumer $i$ is classified into market segment $s$ (zero else), where $P_{i s} \in\{0,1\}$ and $\sum_{S=1}^{S} P_{i s}=1 \forall_{i}$ (for nonoverlapping segments) or $0<\sum_{s=1}^{S^{s}} P_{i s} \leq S$ (for overlapping segments), $\underline{P}=\left(\left(P_{i s}\right)\right)$, and $\varepsilon_{i j t}=$ error (deterministic).

As with all MDU models, there is a portion of the model structure that represents (squared) distance between brands $(\underline{X})$ and ideal points $(\underline{Y})$ in equation 1 above, which spatially portrays brands and segment ideal points in a common $R$ dimensional space (this is often denoted as the "group joint space" as it is typically represented without applying the $a_{t}$ or $\left.b_{t}\right)$. The (squared) distance between a particular brand and segment ideal point is inversely related to the preference exhibited by a segment for a particular brand. As with other metric MDU procedures (DeSarbo and Rao 1986), a linear transformation of this group space via $\underline{a}$ and $\underline{b}$ is included to account for the effects of contexts. These additive and multiplicative constants reflect any level increase or decrease in preference across contexts. For example, all brands may be more/less preferred in some contexts across market segments. In addition, such constants are useful in modeling interval scales in metric analyses whose origin is indeterminate. When all $a_{t}$ are positive, all dimensions in the jointspace plot of $\underline{X}$ and $\underline{Y}_{t}$ can be stretched/shrunk accordingly by context as a way of modeling salience by context. When unconstrained, such $a_{t}$ can allow for ideal points or antiideal points (see Carroll 1980) that are often much more difficult to interpret. Thus, such $a_{t}$ allow for the construction of separate joint spaces by context as a function of linear multiplicative transformations of the group space. Again, the particular model structure in equation 1 is in line with spatial developments in three-way metric multidimensionalscaling methods in terms of parsimoniously accommodating the representation of each way of the input data array (as opposed to having to analyze each context array separately and having to deal with the possibility of different numbers, type of dimensions, or segments).

Our procedure allows for either partitions or overlapping segments via $P$. Even though the current model accounts for consumer heterogeneity by incorporating consumer seg- 
ments and differences in preferences over context, the presence of overlapping segments and their interpretation can provide very useful additional information about the structure of the preference. In the current model, individuals that belong to the same segment have a similar preference structure, and, depending upon the specific application, this can often be interpreted in terms of similar salient goals. For instance, nonoverlapping segments are often identified as grouping individuals who are price conscious and separately grouping those who exhibit more of a variety-seeking tendency. In the nonoverlapping case, the model suggests that individuals in the same cluster show this pattern of preference over different contexts. There is, however, evidence that individuals have multiple salient goals and that until they are forced to make an actual choice, they may keep a preference for multiple options that are associated with different goals (Ratneshwar, Pechmann, and Shocker 1996). Ratneshwar et al. argue that it is even more so if the goals are conflicting. Because the type of data collected here involves preferences and not choices, individuals can simultaneously prefer options that are associated with different goals that might seem conflicting. This is because by asking them for preferences and not choices, they do not need to narrow matters down to a single option.

It is thus possible to interpret the presence of overlapping segments (when individuals are members of multiple clusters) as an additional layer of heterogeneity. The preference of individuals who are in multiple clusters can be thought of as representative of multiple orientations in terms of the goals that they pursue. For example, an individual may express both variety-seeking and price-conscious tendencies over the different usage intentions, even though seeking variety might often involve trying products that are somewhat more expensive. Thus, the use of overlapping segments in our model permits the identification of dependencies between the preferences of different segments and, at the same time, provides a parsimonious representation of what these preference tendencies are. If using overlapping segments does not significantly improve variance accounted for (VAF) over nonoverlapping segments, this would provide some empirical evidence that few individuals exhibit conflicts in their preferences that would correspond to such different tendencies. Note that overlapping segments encompasses nonoverlapping segments as a special nested case when there is no dependency between the segments (Chaturvedi et al. 1997) and is therefore a more general segmentation scheme. The case for overlapping market segments in marketing has otherwise been well established by Arabie et al. (1981), Chaturvedi et al. (1997), DeSarbo, Oliver, and Rangaswamy (1989), Srivastava, Alpert, and Shocker (1984), Wedel and Kamakura (2000), and Wedel and Kistemaker (1989) as a way of dealing with consumer heterogeneity. In addition, note that the methodology can fit models with a single set of ideal points per derived market segment as well as multiple ideal points per market segment.

Thus, we wish to produce a joint space of brands $(\underline{X})$, a series of ideal points $\left(\underline{Y}_{t}\right)$ by market segment and context/ situation, context-specific weights $(a)$ and additive constants $(b)$, and the classification matrix $(\bar{P})$ simultaneously, given the observed data $\Delta$ and user-specified values of the number of market segments $(S)$ and the number of dimensions $(R)$. Unlike some MDU two-way approaches that involve separate analysis by this third data mode (context/situations), we assume the same number and composition of market segments (although this premise can be formally tested by applying our procedure as a two-way method to each situation/context separately and comparing fit values). Here, unlike in Lee et al. (2002), $T$ is given and known. We later relax some of these model assumptions in terms of a more general framework presented in the discussion section of this manuscript.

Visually, in $R=2$ dimensions with $J=10$ brands labeled A-J, $T=4$ contexts/situations, and $S=3$ market segments, figure 1 illustrates a hypothetical group jointspace solution derived by our proposed methodology. Here, $Y_{s}^{t}$ is the notation used to represent the ideal point location for segment $s$ in context/situation $t$. As shown in this illustration, segment 1's four ideal points all fall into the first quadrant and do not vary all that much over the four contexts/situations with respect to their location. Preference appears to be highest for brands $\mathrm{B}, \mathrm{G}$, and $\mathrm{F}$, depending upon the context/situation. Notice the differences when comparing segment 1 here to the remaining two market segments. Segment 2 has its four ideal points located in quadrants 2 , 3 , and 4, as does segment 3. For the members of segment 2 , brands $\mathrm{A}$ and $\mathrm{E}$ are seemingly most preferred in context/ situation 1 , brands D and I, in context/situation 2, and brands

\section{FIGURE 1}

\section{A HYPOTHETICAL SOLUTION IN $R=2$ DIMENSIONS, $T=4$ CONTEXTS, AND $S=3$ SEGMENTS}

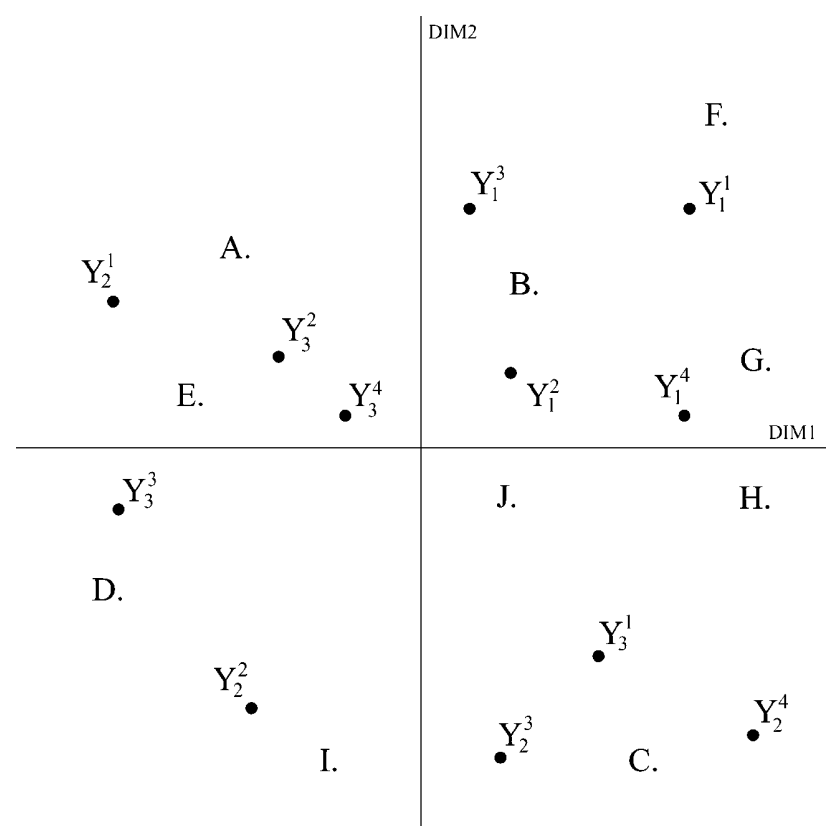


$\mathrm{C}, \mathrm{H}$, and $\mathrm{J}$, in contexts/situations 3 and 4 . For segment 3 , brands $\mathrm{C}, \mathrm{H}$, and $\mathrm{J}$ are most preferred in context/situation 1, brands $\mathrm{A}$ and $\mathrm{E}$, in contexts/situations 2 and 4, and brands $\mathrm{E}$ and $\mathrm{D}$, in context/situation 3 .

We thus posit an ideal point or unfolding MDU display of the structure underlying the preference data while simultaneously classifying the consumers into market segments, allowing for either partitions or overlapping memberships, as well as for single or multiple ideal points at the segment level. Like traditional unfolding MDU models (DeSarbo and Rao 1986), the derived ideal point represents the point of maximum utility or preference for a given segment in a given consumptive context/situation. However, unlike traditional MDU models, we estimate such ideal points per derived market segment. The proposed methodology is much more parsimonious than the traditional approaches and entails the estimation of much fewer parameters given the segment-level estimation.

\section{Estimation}

Given $\Delta=\left(\left(\left(\Delta_{i j t}\right)\right)\right)$ and values of $S$ and $R$, we wish to estimate $\bar{a}=\left(a_{t}\right), \underline{b}=\left(b_{t}\right), \underline{P}=\left(\left(P_{i s}\right)\right), \underline{X}=\left(\left(X_{j r}\right)\right)$, and $\underline{Y}=\left(\left(\left(Y_{s r t}^{-}\right)\right)\right)$so as to minimize

$$
\Phi=\sum_{i=1}^{N} \sum_{j=1}^{J} \sum_{t=1}^{T} \lambda_{i j t}\left(\Delta_{i j t}-\hat{\Delta}_{i j t}\right)^{2},
$$

where $\lambda_{i j t}=$ a user-specified weight for the $i, j, t$ observation, and

$$
\hat{\Delta}_{i j t}=\hat{a}_{t} \sum_{s=1}^{S} \hat{P}_{i s} \sum_{r=1}^{R}\left(\hat{X}_{j r}-\hat{Y}_{s r t}\right)^{2}+\hat{b}_{t} .
$$

The $\lambda_{i j t}$ provides a useful way of accommodating missing data (one can set $\lambda_{i j t}=0$, depending upon the specific causes of such missing data) as well as weighing the observations as in GENFOLD2 (general unfolding analysis, version 2; DeSarbo and Rao 1986) to prevent degenerate MDU solutions (noninformative solutions where the brands and ideal points may be grossly separated-see DeSarbo and Rao 1986). We devise an alternating least-squares iterative estimation procedure involving five stages, all of which conditionally minimize expression 2. Appendix A, available in the online version of $J C R$, describes the technical aspects of the various estimation stages of the proposed clusterwise unfolding methodology. Given the nonlinear nature of the underlying model and objective function, convergence to globally optimum solutions is not guaranteed (as is the case with all other MDU and alternating leastsquares approaches); all existent MDU approaches potentially suffer from problems of local optimum solutions. Multiple runs using different starting values for the parameters are thus recommended. Appendix B, available in the online version of $J C R$, presents the results of a Monte Carlo sim- ulation designed to test the performance of the estimation algorithm as a number of aspects of the data, model, and error are experimentally varied. As shown, the estimation procedure is quite robust.

\section{Model Options}

Given the full model in equation 1 , there are a number of different nested models that we can estimate through the prudent use of restrictions and constraints. For example, the procedure can be used to accommodate either internal ( $X$ is estimated) or external ( $X$ is given and fixed) analyses. Variable $a$ can be restricted to provide a variety of different specifications. For example, $a=1$ can force the group space solution to apply to all contexts. Alternatively, constraining $a_{t}>0$ eliminates the problem of potential antiideal points. The proposed procedure can estimate separate segment-level ideal points by context $\left(Y_{s r t}\right)$ or estimate one common set of ideal points across all contexts $\left(Y_{s r}\right)$. Also, one can estimate partitions or overlapping market segments in $P$.

$\bar{M}$ odel selection in such deterministic MDU models is accommodated through the use of scree plots, incremental search procedures, model diagnostics, and subsequent interpretation of the results. Unlike well-defined parametric models with rigorous distribution theory in place (e.g., multiple regression), there are no parametric model selection tests that can be reliably applied in this scenario. In fact, even with parametric MDU models, such model selection tests are mere heuristics (e.g., AIC [Akaike's information criterion], BIC [Bayesian information criterion], CAIC, ICOMP [informational complexity criterion], etc.) that often render different decisions. In addition, from a technical perspective, higher dimensional/segment MDU solutions are not truly nested with lower dimensional/segment ones.

\section{AN ILLUSTRATION: OTC ANALGESICS MARKET AND DIFFERENTIAL CONTEXT EFFECTS}

\section{OTC Analgesics Market: A Summary}

OTC internal analgesics are orally ingested pain-relieving medications that can be obtained "over the counter," that is, without a doctor's prescription. They are used by $82 \%$ of the population, with headaches and muscle or body aches as the primary uses. There are two main types of OTC analgesics: acetaminophen (brand example: Tylenol) and nonsteroidal anti-inflammatory drugs (NSAIDs), which consist of aspirin (Bayer), ibuprofen (Advil, Motrin, and $\mathrm{Nu}-$ prin), naproxen sodium (Aleve), and ketoprofen (Orudis KT) brands. Some OTC analgesics combine analgesic types as ingredients. Excedrin, for example, contains both aspirin and acetaminophen (American Academy of Family Physicians 2005; MedicineNet 2005). In 2004, consumers purchased $\$ 2.1$ billion worth, or approximately 404.4 million units, of OTC analgesics at supermarkets, grocery stores, conve- 
nience stores, drug stores, and other mass merchandisers (Mintel International Group 2005).

According to the National Center for Health Statistics, the most common use or goal of OTC analgesics is pain relief, particularly relief from headaches or migraines, lower back pain, neck pain, muscle pain, and face or jaw pain. In addition to pain relief, people use analgesics to treat cold, flu, and fever symptoms; to alleviate menstrual discomfort; to curb arthritis and rheumatism pain; to aid sleep; and to prevent the occurrence of heart attacks or strokes (Mintel International Group 2005). The frequency and reasons for taking an OTC analgesic vary along demographic lines (e.g., age). In one survey, respondents in the 18-24 age range reported using some form of nonprescription analgesic an average of five times during the previous 30 days, a somewhat lower number than the overall average of nine times for all adults. Young adults report using OTC analgesics mainly for cold/flu and headache relief, whereas older adults report using OTC pain medicines mostly for arthritis relief and heart attack or stroke prevention.

The most popular OTC general pain relief brands, as measured by market share, are Tylenol, Advil, Aleve, Bayer, Motrin, and Excedrin. Specialized analgesics like Tylenol PM, Tylenol Arthritis, and Excedrin Migraine occupy small market niches. Generic OTC analgesics accounted for $24.5 \%$ of sales during the 52-week period that ended on October 31, 2004 (cf. Lazich 2004). Other brands, including Anacin, Nuprin, Bufferin, St. Joseph, and Vanquish, have a combined market share of $16.9 \%$ (Longe 2005).

Given the highly competitive nature of the OTC analgesic markets, it comes as no surprise that manufacturers seek to differentiate their brands from competitor brands. Some distinction is achieved simply because different analgesic types operate differently for different types of pain. Acetaminophen works on the parts of the brain that receive "pain messages" (American Academy of Family Physicians 2005). NSAIDs work by reducing the body's production of prostaglandins, which cause pain. Both acetaminophen and NSAIDs reduce fever, but only NSAIDs can reduce inflammation and swelling. NSAIDs, but not acetaminophen, are known to cause stomach irritation. Not surprisingly, Wyeth touts the pain-relieving and fever-reducing aspects of Advil, and McNeil advertises Tylenol as the analgesic that is gentle on the stomach. Thus, OTC analgesic effectiveness varies with the type of malady treated.

\section{Study}

A survey was conducted with a sample of 166 current upper-level undergraduate business students at Pennsylvania State University to measure the perceived effectiveness for various major brands of existing OTC analgesic pain relievers. Initially, approximately 10 students were interviewed qualitatively (in stage 1) about how often they use OTC analgesics, the goals or maladies for which they use such medications, and analgesic brand familiarity. Later (after pretesting the questionnaire and subsequent revisions), all students were presented (in stage 2) with 11 major ex- isting OTC analgesic brands that were available in the five major pharmacies and retail outlets in the immediate university area: Advil, Aleve, Anacin, Bayer, Bufferin, Excedrin, Motrin, Nuprin, St. Joseph Aspirin, Tylenol, and Vanquish. Note that these 11 brands account for over $90 \%$ of the nongeneric analgesic OTC market share.

The students were presented color photographs of each brand and its packaging, together with price, number of tablets, recommended dose, price per dose, and all the information provided by the manufacturer found on the packaging. Each respondent was requested to read this information and make personal perceived effectiveness judgments for usage of each of the 11 brands on a four-point scale for treating four different goals/maladies/situations as well as overall effectiveness (used as a benchmark as in DeSarbo and Carroll [1981a, 1981b, 1985] and Green and Rao [1972]). The four maladies, or goals, considered included headache, muscle aches, colds/flu/fever, and backaches - the major maladies listed on the various package labels that were of relevance to this youthful audience (as determined by a literature review on the product class and stage 1 interviewing). We also asked for overall effectiveness. More specifically, the survey asked participants to evaluate OTC analgesics sequentially, one by one. The participants were not asked to jointly compare the products. They were also not asked to indicate brand effectiveness for the analgesics they evaluated in comparison to each other. Rather, they were asked to rate how effective they believed a particular brand would be in meeting a particular goal in treating a particular malady based on their current perceptions, familiarity, personal usage, and the brand information provided to them (package information).

We also collected information concerning the participants' personal usage, psychographic information, demographics, familiarity, and attitudes about OTC analgesics. Given that data were collected in one 45-minute session, arguments for segment or dimension heterogeneity or both are less appropriate here compared to longitudinal studies conducted over several time periods. However, we shall contrast our proposed model results against an alternative (separate MDU analysis by situation followed by separate KMEANS clustering) that accommodates both forms of such heterogeneity.

\section{Proposed Methodology Results}

We took the original five situations (four maladies plus one overall $) \times 166$ students $\times 11$ OTC brands three-way array after the standardization and scale reversal for analysis (see Harshman and Lundy [1984] for a discussion of preprocessing data for three-way multidimensional-scaling methods) with the proposed clusterwise MDU approach. Including overall evaluations as well is consistent with the manner in which DeSarbo and Carroll (1981a, 1981b, 1985) and Green and Rao (1972) perform their three-way analyses in multidimensional scaling by including overall evaluations. This is often done to establish a benchmark in comparison to the situations tested, as well as to examine the basis upon which an overall judgment is made as a function of the 
potential usage situations a consumer is faced with. The analysis was run for both overlapping and nonoverlapping solutions, as well as for single versus multiple ideal points (four different models) for all identifiable ( $S$ [segments] $\geq R$ [dimensions] as in latent class multidimensional-scaling models) combinations of $S$ and $R=1, \ldots, 5$. Table 1 presents the sum of squared error (SSE) and VAF fit measures for all four models run. The $\lambda_{i j t}$ were set at one for all nonmissing data cells and zero for missing data cells. In order to gain some insight into model selection, table 2 reports the results of an unbalanced ANOVA performed on the logit-transformed VAF measure (to stabilize the variance) using $R, S$, type of segments estimated (labeled overlapping), and type of ideal points estimated (labeled dynamic) as independent factors. As shown, the main effects analysis produces an adjusted $R^{2}=0.937$, with all factors except $R$ highly significant. The eta-square measures provide some information as to the relative importance of such factors, where the number of segments and overlapping segments clearly dominate the analysis, followed by multiple ideal points. Thus, given these results, it does appear that an overlapping segment, multiple-ideal-point representation is best, given that each of these two-level factors is significant. A deeper investigation is provided in tables 3 and 4, where Bonferroni post hoc contrasts are provided for $R$ and $S$ across all four models. In table 3, it is clear from these post hoc contrasts that the dimensionality ceases to significantly improve the logit VAF measure beyond two dimensions. In table 4 , we see that the number of segments beyond three ceases to significantly improve this fit measure as well. Thus, we shall select the $R=2$ dimensions, $S=3$ overlapping segments, multiple-ideal-point model (M4) as the best rep-

TABLE 1

GOODNESS-OF-FIT MEASURES FOR FOUR DISTINCT CLUSTERWISE MULTIDIMENSIONAL UNFOLDING MODELS

\begin{tabular}{|c|c|c|c|c|c|c|c|c|c|}
\hline \multirow[b]{2}{*}{$R$} & \multirow[b]{2}{*}{$S$} & \multicolumn{2}{|l|}{ M1 } & \multicolumn{2}{|c|}{ M2 } & \multicolumn{2}{|l|}{ M3 } & \multicolumn{2}{|c|}{ M4 } \\
\hline & & SSE & VAF & SSE & VAF & SSE & VAF & SSE & VAF \\
\hline 1 & 1 & $8,688.5$ & .002 & $8,688.5$ & .002 & $8,593.4$ & .013 & $8,593.4$ & .013 \\
\hline 1 & 2 & $8,217.6$ & .056 & $7,477.2$ & .141 & $7,630.2$ & .123 & $7,441.6$ & .145 \\
\hline 1 & 3 & $7,339.0$ & .157 & $6,982.5$ & .198 & $7,291.4$ & .162 & $6,885.4$ & .209 \\
\hline 1 & 4 & $7,125.8$ & .181 & $6,790.1$ & .220 & $7,093.5$ & .185 & $6,745.4$ & .225 \\
\hline 1 & 5 & $6,932.7$ & .203 & $6,478.9$ & .255 & $6,921.6$ & .205 & $6,368.0$ & .268 \\
\hline 2 & 2 & $7,620.3$ & .124 & $7,341.6$ & .156 & $7,535.9$ & .134 & $7,290.1$ & .162 \\
\hline 2 & 3 & $7,301.1$ & .161 & $6,864.8$ & .211 & $7,205.1$ & .172 & $6,675.3$ & .233 \\
\hline 2 & 4 & $7,122.5$ & .182 & $6,424.5$ & .262 & $7,076.2$ & .187 & $6,302.9$ & .276 \\
\hline 2 & 5 & $6,892.4$ & .208 & $6,168.4$ & .291 & $6,852.8$ & .213 & $6,055.8$ & .304 \\
\hline 3 & 3 & $7,297.0$ & .161 & $6,775.2$ & .221 & $7,146.2$ & .179 & $6,628.5$ & .238 \\
\hline 3 & 4 & $7,033.2$ & .192 & $6,325.7$ & .273 & $6,939.4$ & .203 & $6,211.1$ & .286 \\
\hline 3 & 5 & $6,879.1$ & .210 & $6,092.2$ & .300 & $6,810.8$ & .217 & $5,893.6$ & .323 \\
\hline 4 & 4 & $7,007.6$ & .195 & $6,325.2$ & .273 & $6,856.6$ & .212 & $6,200.8$ & .287 \\
\hline 4 & 5 & $6,861.3$ & .212 & $6,079.0$ & .301 & $6,728.8$ & .227 & $5,891.4$ & .323 \\
\hline 5 & 5 & $6,837.8$ & .214 & $6,025.8$ & .308 & $6,664.9$ & .234 & $5,868.9$ & .326 \\
\hline
\end{tabular}

TABLE 2

MAIN EFFECTS ANOVA ON LOGIT VARIANCE ACCOUNTED FOR

\begin{tabular}{|c|c|c|c|c|c|c|}
\hline Source & $\begin{array}{l}\text { Type } 3 \\
\text { sum of } \\
\text { squares }\end{array}$ & $d f$ & $\begin{array}{l}\text { Mean } \\
\text { square }\end{array}$ & $F$ & $p$ & $\begin{array}{c}\text { Par- } \\
\text { tial } \\
\eta^{2}\end{array}$ \\
\hline Corrected model & 65.997 & 10 & 6.600 & 88.34 & .000 & .947 \\
\hline Intercept & 119.984 & 1 & 119.984 & $1,606.03$ & .000 & .970 \\
\hline Dynamic & .699 & 1 & .699 & 9.36 & .004 & .160 \\
\hline Overlapping & 2.198 & 1 & 2.198 & 29.42 & .000 & .375 \\
\hline$R$ & .351 & 4 & .088 & 1.17 & .333 & .088 \\
\hline$S$ & 47.573 & 4 & 11.893 & 159.19 & .000 & .929 \\
\hline Error & 3.661 & 49 & .075 & & & \\
\hline Total & 220.871 & 60 & & & & \\
\hline Corrected total & 69.657 & 59 & & & & \\
\hline
\end{tabular}

NoTE.- $R^{2}=.947$; adjusted $R^{2}=.937$.

resentation of the structure of these data (with $\mathrm{SSE}=$ $6,675.3$, VAF $=0.233$ ). As with traditional multidimensional scaling and the heuristics employed for model selection, this result can also be verified by incremental search analysis, as well as the by the clear interpretation of the derived solution.

Figure 2 presents the brand space that was estimated by the proposed methodology (we remove the segment-level ideal points for now). We also calculated correlations of these brand coordinates with many of the various attributes listed on the package and also presented to these students (various ingredients, market share, and price per dose), as is performed in standard multidimensional-scaling property

TABLE 3

BONFERRONI POST HOC MULTIPLE COMPARISONS FOR $R$ MODEL SELECTION

\begin{tabular}{|c|c|c|c|c|c|c|}
\hline \multirow{2}{*}{$\begin{array}{l}R \\
(I)\end{array}$} & \multirow{2}{*}{$\begin{array}{c}R \\
(J)\end{array}$} & \multirow{2}{*}{$\begin{array}{c}\text { Mean } \\
\text { difference } \\
(I-J)\end{array}$} & \multirow[b]{2}{*}{ SE } & \multirow[b]{2}{*}{$p$} & \multicolumn{2}{|c|}{$95 \%$ confidence interval } \\
\hline & & & & & Lower bound & Upper bound \\
\hline \multirow[t]{4}{*}{1} & 2 & $-.900^{*}$ & .092 & .00 & -1.170 & -.631 \\
\hline & 3 & $-1.081^{*}$ & .100 & .00 & -1.375 & -.788 \\
\hline & 4 & $-1.196^{\star}$ & .114 & .00 & -1.533 & -.860 \\
\hline & 5 & $-1.285^{\star}$ & .150 & .00 & -1.725 & -.845 \\
\hline \multirow[t]{4}{*}{2} & 1 & $.900^{*}$ & .092 & .00 & .631 & 1.170 \\
\hline & 3 & -.181 & .104 & .89 & -.488 & .126 \\
\hline & 4 & -.296 & .118 & .16 & -.644 & .052 \\
\hline & 5 & -.385 & .153 & .15 & -.834 & .065 \\
\hline \multirow[t]{4}{*}{3} & 1 & $1.081^{*}$ & .100 & .00 & .788 & 1.375 \\
\hline & 2 & .181 & .104 & .89 & -.126 & .488 \\
\hline & 4 & -.115 & .125 & 1.00 & -.482 & .252 \\
\hline & 5 & -.204 & .158 & 1.00 & -.667 & .260 \\
\hline \multirow[t]{4}{*}{4} & 1 & $1.196^{*}$ & .114 & .00 & .860 & 1.533 \\
\hline & 2 & .296 & .118 & .16 & -.052 & .644 \\
\hline & 3 & .115 & .125 & 1.00 & -.252 & .482 \\
\hline & 5 & -.088 & .167 & 1.00 & -.581 & .404 \\
\hline \multirow[t]{4}{*}{5} & 1 & $1.285^{\star}$ & .150 & .00 & .845 & 1.725 \\
\hline & 2 & .385 & .153 & .15 & -.065 & .834 \\
\hline & 3 & .204 & .158 & 1.00 & -.260 & .667 \\
\hline & 4 & .088 & .167 & 1.00 & -.404 & .581 \\
\hline
\end{tabular}

*Mean difference (based on observed means) is significant at the .05 level. 
TABLE 4

BONFERRONI POST HOC MULTIPLE COMPARISONS FOR $S$ MODEL SELECTION

\begin{tabular}{|c|c|c|c|c|c|c|}
\hline \multirow{2}{*}{$\begin{array}{l}S \\
(I)\end{array}$} & \multirow{2}{*}{$\begin{array}{c}S \\
(J)\end{array}$} & \multirow{2}{*}{$\begin{array}{c}\text { Mean } \\
\text { difference } \\
(I-J)\end{array}$} & \multirow[b]{2}{*}{ SE } & \multirow[b]{2}{*}{$p$} & \multicolumn{2}{|c|}{$95 \%$ confidence interval } \\
\hline & & & & & Lower bound & Upper bound \\
\hline \multirow[t]{4}{*}{1} & 2 & $-3.331^{*}$ & .167 & .00 & -3.823 & -2.839 \\
\hline & 3 & $-3.823^{*}$ & .158 & .00 & -4.286 & -3.359 \\
\hline & 4 & $-4.036^{*}$ & .153 & .00 & -4.485 & -3.587 \\
\hline & 5 & $-4.196^{*}$ & .150 & .00 & -4.637 & -3.756 \\
\hline \multirow[t]{4}{*}{2} & 1 & $3.331^{*}$ & .167 & .00 & 2.839 & 3.823 \\
\hline & 3 & $-.492^{*}$ & .125 & .00 & -.859 & -.125 \\
\hline & 4 & $-.705^{\star}$ & .118 & .00 & -1.053 & -.357 \\
\hline & 5 & $-.866^{\star}$ & .114 & .00 & -1.202 & -.530 \\
\hline \multirow[t]{4}{*}{3} & 1 & $3.823^{*}$ & .158 & .00 & 3.359 & 4.286 \\
\hline & 2 & $.492^{*}$ & .125 & .00 & .125 & .859 \\
\hline & 4 & -.214 & .104 & .46 & -.520 & .093 \\
\hline & 5 & $-.374^{*}$ & .100 & .01 & -.667 & -.081 \\
\hline \multirow[t]{4}{*}{4} & 1 & $4.036^{*}$ & .153 & .00 & 3.587 & 4.485 \\
\hline & 2 & $.705^{\star}$ & .118 & .00 & .357 & 1.053 \\
\hline & 3 & .214 & .104 & .46 & -.093 & .520 \\
\hline & 5 & -.160 & .092 & .86 & -.430 & .109 \\
\hline \multirow[t]{4}{*}{5} & 1 & $4.196^{*}$ & .150 & .00 & 3.756 & 4.637 \\
\hline & 2 & $.866^{\star}$ & .114 & .00 & .530 & 1.202 \\
\hline & 3 & $.374^{\star}$ & .100 & .01 & .081 & .667 \\
\hline & 4 & .160 & .092 & .86 & -.109 & .430 \\
\hline
\end{tabular}

*Mean difference (based on observed means) is significant at the .05 level

fitting in order to aid in the interpretation of the derived dimensions (cf. Green and Rao 1972). Table 5 presents these correlations from which we readily interpret the derived dimensions. The horizontal dimension 1 separates brands with less popular aspirin from the more popular non-aspirinbased products. The vertical dimension 2 separates the brands containing acetaminophen from the nonacetaminophen brands. Note that the brands span all four quadrants of this derived two-dimensional space.

Rather than display the total joint-space result in one plot for all three segments across all five consumptive maladies or situations as in figure 1, in order to simplify the distinctions to be made in the following text, we present figures 3-5, which are the corresponding joint-space solutions estimated for each of the three market segments across the five consumptive situations. Interestingly, we see no evidence from these joint-space plots for a vector model solution, as all estimated ideal points are bounded well within the general range of the brand locations (i.e., no ideal points go off to infinity). Nor do we witness a degenerate MDU solution, despite setting $\lambda_{i j t}=1$ for all nonmissing data values $\left(\lambda_{i j t}=0\right.$ for missing data values) and not employing a GENFOLD2 weighting system. Figure 3 displays the estimated multiple ideal points for segment 1. Overall, there appears to be dominance in perceived effectiveness for Advil and Tylenol; for headaches, Excedrin is clearly deemed most effective. The ibuprofen and naproxen-formulated brands appear most effective for muscle/joint pain and fever/colds/ flu. For backaches, the aspirin brands are most effective. Of the three segments derived, this particular segment appears to most closely follow market information/knowledge as to
FIGURE 2

OVER-THE-COUNTER ANALGESIC BRAND SPACE

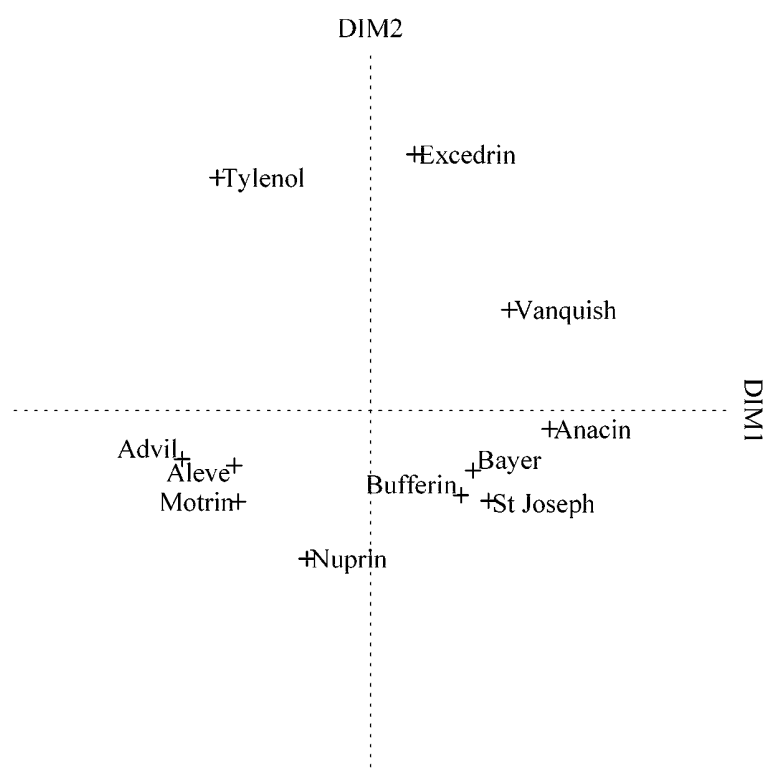

which particular ingredients perform best for different maladies. What clearly distinguishes members of this segment from nonmembers is price sensitivity (members of segment 1 are very price sensitive when it comes to purchasing OTC analgesics). Members of this segment also are not very socially active. We will label this segment as the price-conscious segment. The derived market segments were interpreted by relating the estimated $P$ classification matrix to the demographic, psychographic, and usage information also collected in the survey.

Figure 4 displays this configuration of multiple ideal points for segment 2. Overall, dominance in perceived effectiveness appears to be for the aspirin-based brands, as it is for muscle/joint pain. For headaches, the ibuprofen and naproxen brands are most effective. For fever/colds/flu, movement is toward the acetaminophen brands. Aspirin brands are most effective for backaches. Members of this segment are more likely to be student athletes, believe they get sick more often, are germ conscious, and wake up each

TABLE 5

CORRELATIONS BETWEEN BRAND COORDINATES AND ATTRIBUTES

\begin{tabular}{lrr}
\hline \hline & Dimension 1 & Dimension 2 \\
\hline Price/dose & .747 & .061 \\
Ibuprofen (mg) & -.605 & -.450 \\
Aspirin (mg) & .855 & .120 \\
Acetaminophen (mg) & -.070 & .952 \\
Market share & -.807 & .416 \\
\hline
\end{tabular}


FIGURE 3

DERIVED JOINT SPACE FOR SEGMENT 1

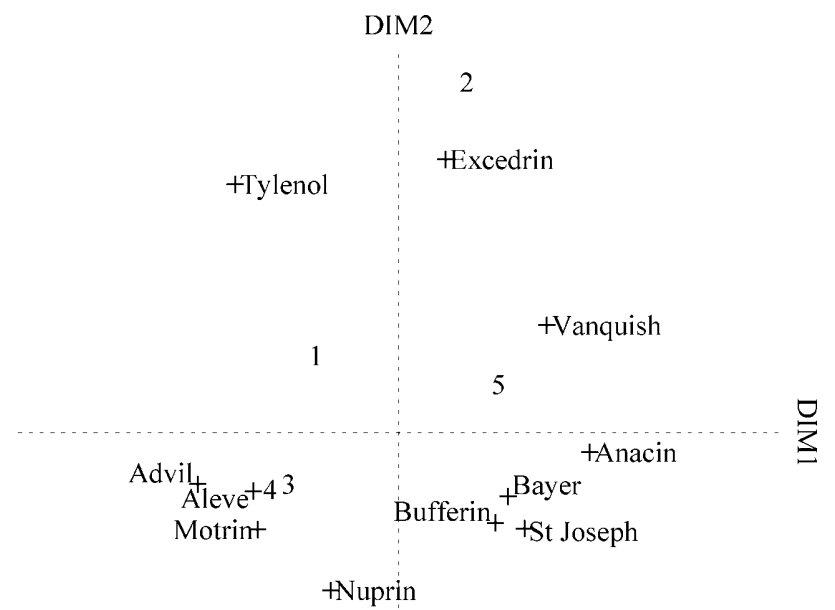

NoTE.-Situation key is $1=$ overall, $2=$ headaches, $3=$ muscle/joint pain, $4=$ fever/colds/flu, and $5=$ backaches.

morning sore and achy more frequently than nonmembers. We can label this segment as the athletic segment.

Figure 5 displays the estimated joint space for segment 3. Overall, dominance in perceived effectiveness is for the nonaspirin brands. The ibuprofen and naproxen brands are most effective for headache relief, whereas the acetaminophen brands are most effective for muscle/joint pain. The aspirin brands are effective for fever/colds/flu, and Tylenol is effective for backaches. Members of this segment are more likely to dislike taking any form of medicine, do not like experimenting with OTC medications when ill, are less likely to comply with doctors prescriptions when ill, dislike fraternity/sorority parties, do not use OTC analgesics for hangovers, and tend to be younger than nonmembers. We label this segment as an antimedication segment.

One very interesting finding here, as aptly illustrated in all three figures, concerns the comparative locations for the ideal points for the overall effectiveness context versus the specific context-dependent ideal points for each of the three derived market segments. There are rather pronounced differences seen with respect to the ideal points associated with this overall benchmark compared to the situation/maladyspecific ideal points for each of the segments. This suggests a potential masking of the results when an overall preference/effectiveness measure is collected alone in consumer studies where context/situation is important.

In terms of the overlapping segment sizes, $75.3 \%$ of the sample were members of segment $1 ; 56.6 \%$, of segment 2 ; and $49.4 \%$, of segment 3 . Roughly $17 \%$ of the sample were members of all three segments, $46 \%$ were allocated to two segments, while $36 \%$ of the sample were allocated just to a single segment. Figure 6 depicts this extensive overlap in Venn diagram form. The presence of overlap indicates that some students may try to juggle multiple objectives that conflict when establishing their preference. There are $22.3 \%$ of the students who are exclusively price conscious and antimedication. Although these students generally avoid medications, they prefer to buy the cheapest OTC options available when needed. In contrast, $19.9 \%$ of the students belong to both the athletic and the price-conscious segments. This might indicate that as student athletes, they are on a budget and try to manage by focusing on the cheapest medicine that would serve their needs. Given this amount of overlap, it is not surprising that the overlapping solutions dominated the nonoverlapping solutions for this application. This suggests more complex and extensive student response heterogeneity with respect to these effectiveness perceptions.

\section{Comparative Analysis}

As we have alluded to in the introduction, there are alternative approaches that can be employed to obtain segment-level joint spaces and classification into market segments via the sequential application of multiple procedures. Unfortunately, comparison with the Lee et al. (2002) approach here is impossible as their method requires choice data and switching matrices, which are not available in most

FIGURE 4

DERIVED JOINT SPACE FOR SEGMENT 2

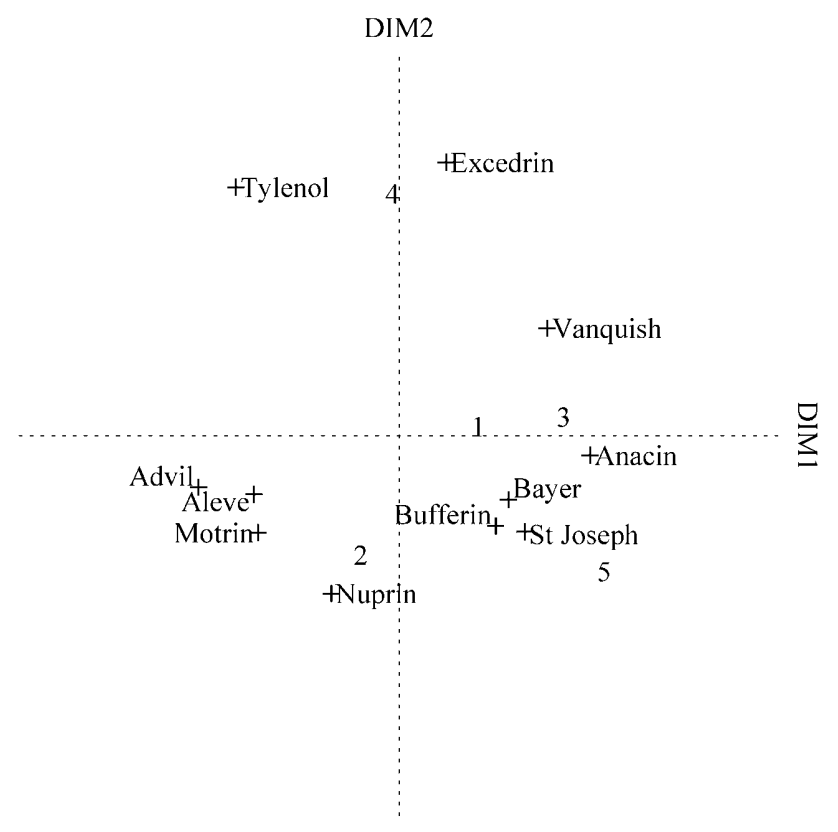

NOTE.-Situation key is $1=$ overall, $2=$ headaches, $3=$ muscle/joint pain, $4=$ fever/colds/flu, and $5=$ backaches. 
FIGURE 5

DERIVED JOINT SPACE FOR SEGMENT 3

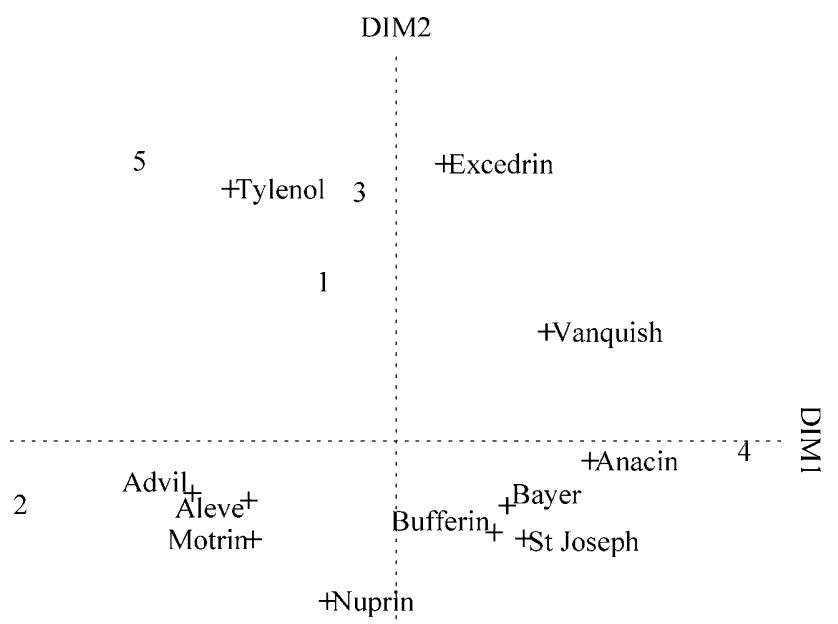

NoTE.-Situation key is $1=$ overall, $2=$ headaches, $3=$ muscle/joint pain, $4=$ fever/colds/flu, and $5=$ backaches.

consumer survey data of this type. We then wish to explore the alternative goodness of fits obtained in one of the most flexible approaches in accommodating both segment and dimension heterogeneity: applying two-way unfolding to each context separately and then clustering each set of resulting ideal points separately. As mentioned, this two-stage alternative has the advantage of accommodating preference, dimension, and segmentation heterogeneity but at a cost of higher parameterization, potentially incongruent results from context to context, and ad hoc decisions with respect to clustering. In this effort, we applied GENFOLD3, a metric version of GENFOLD2 (DeSarbo and Rao 1986), to the same standardized data (by malady/situation) used in the proposed procedure (five separate analyses). We saved the derived configurations from that set of analyses, and then conducted five separate KMEANS clustering analyses (one per set of estimated ideal points). Here, two dimensions were indicated as most parsimonious from all five GENFOLD3 analyses, and three market segments were indicated as "best" for four of the five contexts in KMEANS. Using the resulting classifications and mean ideal point values, together with the five brand configurations and additive and multiplicative constants, we then calculated predicted utility values and the two goodness-of-fit measures (SSE and VAF). Surprisingly, we obtained $\mathrm{SSE}=25,587.3$ and $\mathrm{VAF}=$ 0.023 , which are much worse than the values for the proposed procedure, especially considering the much larger number of parameters being estimated. A portion of this poor fit is due to the fact that the two disjoint procedures optimize totally different loss functions, unlike the simultaneous nonlinear alternating least-squares-based procedure, which solves for both the joint space and segmentation simultaneously. Another contributing factor to this poor fit is due to the fact that KMEANS is restricted to solve for only partitions and not overlapping segments. Finally, the $\lambda_{i j t}$ in GENFOLD3 were calculated as a function of the order of the preference data as degenerate solutions were observed by setting them equal to one for nonmissing observations. These weights tend to result in recovering the higher effective brands much better than the lower effective ones. Thus, the additional heterogeneity (and number of parameters) accounted for by this two-step method does not appear to compensate for the problems associated with using disjointed methods in sequence, which optimize different criteria for this application.

\section{DISCUSSION}

We have presented a new methodology for the estimation of multiple ideal points. After reviewing the extensive literature on context and situational effects in marketing and consumer research, a new clusterwise MDU procedure was technically described for use where there are known discrete contexts, time periods, goals, situations, uses, and so on. An application of this proposed procedure was given with respect to a student study conducted to examine OTC analgesics' effectiveness for various consumptive goals involving different maladies. We applied the proposed clusterwise MDU procedure and derived an $R=2$ dimensions and $S=3$ market segments solution and described the joint space and multiple ideal points derived by segment across

\section{FIGURE 6}

\section{DERIVED OVER-THE-COUNTER ANALGESICS OVERLAPPING MARKET SEGMENTS}

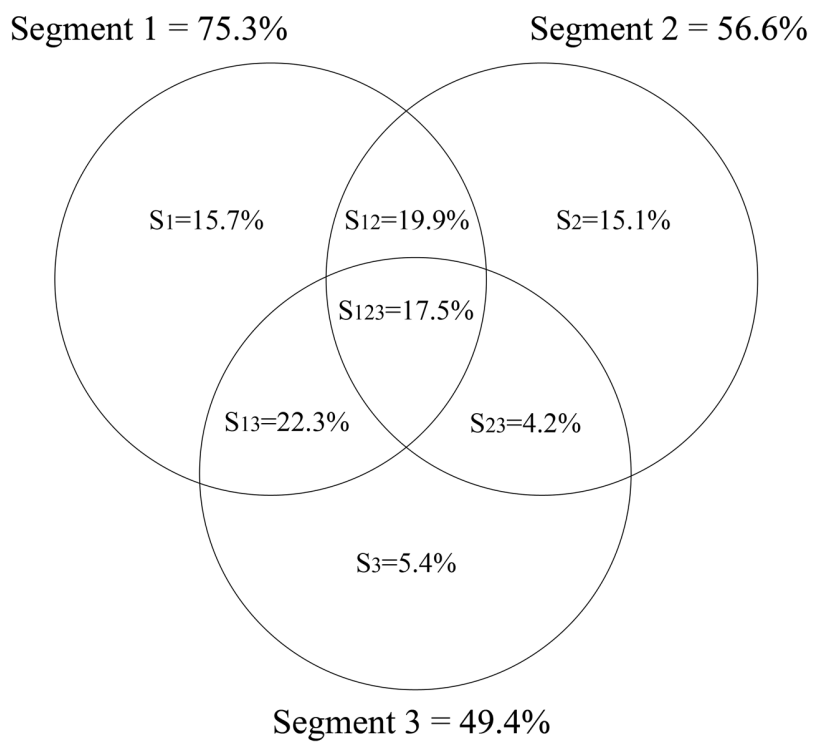


the various maladies. We fit four different models and demonstrated how an overlapping clusterwise solution with multiple ideal points dominated other simpler clusterwise MDU solutions derived from the same methodology, as well as a two-stage solution obtained by the sequential application of a two-way MDU procedure and then a cluster analysis-all within context.

Further research is needed in this area. First, a fully nonmetric version would be useful for applications involving ordinal scales of measurement. Second, research on developing better heuristics for model selection (selecting $R$ and $S$ ) is desirable, especially model selection heuristics, which trade off model fit versus model complexity. Third, the implementation of linear restrictions on point coordinates as in GENFOLD2 (DeSarbo and Rao 1986) would prove fruitful, especially for optimal positioning applications. Fourth, applying the procedure across a multitude of different marketing applications and data would better reveal the utility of such a methodology. In particular, since the methodology can accommodate any form of two- or three-way dominance data, applications in consumer research involving longitudinal purchase data (e.g., scanner/panel data), behavioral intentions data (e.g., prescriptions from doctors for various brands of drugs in a designated product class), full-profile conjoint analyses (i.e., respondents $\times$ profiles), and so on, would prove useful in further demonstrating the utility of this approach. Fifth, generalizing the model to include context and dimension weights $\left(W_{r t}\right)$ in place of the multiplicative constants $\left(a_{t}\right)$ would make the procedure more flexible as in INDSCAL (individual differences scaling; Carroll 1972) and three-way unfolding (DeSarbo and Carroll 1985). Sixth, deriving confidence bands around estimated segment ideal points and brand locations would be desirable (cf. Kiers and Groenen [2006] for a survey of various resampling methods for performing this for deterministic multidimensional scaling procedures). Seventh, extending the model to accommodate preference, perceptual, and segment heterogeneity would prove to be an ambitious research goal. One of the limitations of the proposed methodology is that there is an explicit restriction on the membership indicator matrix $\underline{P}$ to be constant across consumptive situations. For example, one such generalization could employ the following framework. Using the previous notation developed, let $s_{t}=$ $1, \ldots, S_{t}$ market segments in situation $t$ (unknown) and $r_{t}=1, \ldots, R_{t}$ dimensions in situation $t$ (unknown).

One could then model the observed reverse scaled preference data as

$$
\Delta_{i j t}=a_{t} \sum_{s_{t}=1}^{S_{t}} P_{i s_{t}} \sum_{r_{t}=1}^{R_{t}}\left(X_{j r_{t}}-Y_{s r_{t}}\right)^{2}+b_{t}+\varepsilon_{i j t},
$$

where $X_{j r_{t}}=$ the $r_{t}$ th coordinate for brand $j$ in situation $t$, $Y_{s r_{t}}=$ the $r_{t}$ th coordinate of the ideal point for market segment $s$ in situation $t, a_{t}=$ a multiplicative constant for situation $t, b_{t}=$ an additive constant for situation $t$, and $P_{i s_{t}}=1$ if consumer $i$ is classified into market segment $s_{t}$ in situation $t$ (zero else), where $P_{i s_{t}} \in\{0,1\}$ and $\sum_{s_{t}=1}^{S_{t}} P_{i s_{t}}=$ $1 \forall_{i}$ (for nonoverlapping segments) or $0<\sum_{s_{t}=1}^{S_{t}} P_{i s_{t}} \leq S_{t}$ (for overlapping segments), and $\varepsilon_{i j t}=$ error. This general framework or saturated model would accommodate a number of special features that no other ordinary or clusterwise multidimensional-scaling procedure handles. One, the general model exposition in equation 4 accommodates applications where the dimensionality may differ by context or situation. This involves two different aspects: $(a)$ perceptual heterogeneity over situations in which the brand space may involve different dimensionality or the same number of dimensions but different interpretive dimensions by situation and $(b)$ preference heterogeneity over situations where segment-level utility functions are allowed to shift from segment to segment (e.g., multiple ideal points). Two, this general model also handles applications involving segment heterogeneity by situations in which either the number of segments change from one situation to another or the same number of segments exists but the memberships alter by situation. Note that allowing all three forms of heterogeneity to exist in this general model simultaneously is equivalent to applying the existing proposed two-way clusterwise model on each situational data slice separately. Given the identification problems with some of the parameters in equation 4, additional research is needed to accommodate various constrained versions of this saturated model with accompanying model selection heuristics.

Finally, extending this segment-level model to the individual level would be a useful generalization of this spatial model. Here, the underlying model could be of the following form:

$$
\Delta_{i j t}=\sum_{r=1}^{R} W_{r t}\left(X_{j r}-Y_{i r t}\right)^{2}+b_{t}+\varepsilon_{i j t}
$$

without market segment estimation. Such a model would be highly parameterized, given the huge amount of parameters, and would extend the DeSarbo and Carroll (1985) three-way unfolding model to multiple ideal points.

\section{REFERENCES}

American Academy of Family Physicians (2005), "Pain Relievers: Understanding Your OTC Options," http://familydoctor.org/.

Arabie, Phipps, J. Douglas Carroll, Wayne S. DeSarbo, and Jerry Wind (1981), "Overlapping Clustering: A New Method for Product Positioning," Journal of Marketing Research, 18 (August), 310-17.

Belk, Russell W. (1974), “An Exploratory Assessment of Situational Effects in Buyer Behavior," Journal of Marketing Research, 11 (2), 156-63.

(1975), "Situational Variables and Consumer Behavior," Journal of Consumer Research, 2 (3), 157-64.

(1979), "A Free Response Approach to Developing Product-Specific Taxonomies," in Analytical Approaches to Product and Marketing Planning, ed. Allan D. Shocker, Cambridge, MA: Marketing Science Institute. 
Bettman, James R., Mary Frances Luce, and John W. Payne (1998), "Constructive Consumer Choice Processes," Journal of Consumer Research, 25 (3), 187-217.

Carlson, Kurt A. and Samuel D. Bond (2006), "Improving Preference Assessment Limiting the Effect of Context through Pre-exposure to Attribute Levels," Management Science, 52 (3), 410-21.

Carroll, J. Douglas (1972), "Individual Differences and Multidimensional Scaling," in Multidimensional Scaling, ed. A. Kimball Romney and Sara Beth Nerlove, New York: Seminar.

- (1980), "Models and Methods for Multidimensional Analysis of Preferential Choice (or Other Dominance) Data," in Similarity and Choice, ed. Ernest D. Lantermann and Hubert Feger, Vienna: Huber.

Chaturvedi, Anil, J. Douglas Carroll, Paul E. Green, and John A. Rotondo (1997), "A Feature Based Approach to Market Segmentation via Overlapping K-Centroids Clustering," Journal of Marketing Research, 34 (3), 370-77.

DeSarbo, Wayne S. and J. Douglas Carroll (1981a), "Three-Way Metric Unfolding," in Proceedings of the ORSA/TIMS Market Measurement Conference, ed. John W. Keon, Providence, RI: Institute of Management Sciences, 157-83.

- (1981b), "Three-Way Unfolding and Situational Dependence in Consumer Preferences Analysis," in The Changing Marketing Environment: New Theories and Applications, ed. K. Bernhardt, Chicago: American Marketing Association, 321-25.

_ (1985), "Three-Way Metric Unfolding via Weighted LeastSquares," Psychometrika, 50 (September), 275-300.

DeSarbo, Wayne S., Richard L. Oliver, and Arvind Rangaswamy (1989), "A Simulated Annealing Methodology for ClusterWise Linear Regression," Psychometrika, 54 (4), 707-36.

DeSarbo, Wayne S. and Vithala R. Rao (1986), "A Constrained Unfolding Model for Product Positioning," Marketing Science, 5 (1), 1-19.

Dubow, Joel S. (1992), "Occasion-Based vs. User-Based Benefit Segmentation: A Case Study," Journal of Advertising Research, 32 (March-April), 11-18.

Green, Paul E. and Vithala R. Rao (1972), Applied Multidimensional Scaling, Hinsdale, IL: Dryden.

Gupta, Sunil and Joel H. Steckel (1993), "Preference Aggregation and Repeat Buying in Households," Marketing Letters, 4 (4), 321-36.

Hagerty, Michael R. (1980), “A Deterministic Model of Consumer Choice," in Advances in Consumer Research, Vol. 7, ed. Jeny C. Olson, Ann Arbor, MI: Association for Consumer Research, 129-33.

Harshman, Richard A. and Margaret E. Lundy (1984), "Data Preprocessing and the Extended PARAFAC Model," in Research Methods for Multimode Data Analysis, ed. Henry G. Law, Conrad W. Snyder, John A. Hattie, and Roderick P. McDonald, New York: Praeger, 216-84.

Holbrook, Morris B. (1984), "Situation-Specific Ideal Points and Usage of Multiple Dissimilar Brands," in Research in Marketing, Vol. 7, ed. Jagdish N. Sheth, Greenwich, CT: JAI Press, 93-131.

Kahn, Barbara E., Donald G. Morrison, and Gordon P. Wright
(1986), "Aggregating Individual Purchases to the Household Level," Marketing Science, 5 (Summer), 260-68.

Kiers, Henk A. L. and Patrick J. F. Groenen (2006), "Visualizing Dependence of Bootstrap Confidence Intervals for Methods Yielding Spatial Configurations," in Studies in Classification, Data Analysis, and Knowledge Organization, ed. Sergio Zani, Andrea Ceroli, Marco Riani, and Maurizio Vichi, Berlin: Springer, 119-26.

Laurent, Gilles (1978), "A Study of Multiple Variant Consumption for Frequently Purchased Consumer Items," unpublished dissertation, Sloan School of Management, Cambridge, MA 02142 .

Lazich, Robert S., ed. (2004), Market Share Reporter, 2005, Vol. 2, Farmington Hills, MI: Gale Group.

Lee, Jack K. H., Karunakaran Sudhir, and Joel H. Steckel (2002), "A Multiple Ideal Point Model: Capturing Multiple Preference Effects from within an Ideal Point Framework," Journal of Marketing Research, 39 (1), 73-86.

Longe, Jacqueline (2005), The Gale Encyclopedia of Medicine, Detroit: Gale Group.

MedicineNet (2005), "Pain Management_Pain Medication and Overthe-Counter (OTC) Drugs," http://www.medicinenet.com/.

Mintel International Group (2005), "OTC Analgesics-US-May 2005," report, Mintel International Group, Chicago, http:// reports.mintel.com/sinatra/reports/index/\&letter $=15 /$ display/ id $=226649$ \&anchor $=$ a226649.

Ratneshwar, Srinivasan, Cornelia Pechmann, and Allan D. Shocker (1996), "Goal-Derived Categories and the Antecedents of across-Category Consideration," Journal of Consumer Research, 23 (December), 240-50.

Simon, Herbert A. (1955), "A Behavioral Model of Rational Choice," Quarterly Journal of Economics, 69 (February), 99-118.

- (1990), "Invariance of Human Behavior," Annual Review of Psychology, 41 (January), 1-19.

Srivastava, Rajendra K., Mark I. Alpert, and Allan D. Shocker (1984), "A Customer Oriented Approach for Determining Market Structures," Journal of Marketing, 48 (2), 32-45.

Srivastava, Rajendra K., Allan D. Shocker, and George S. Day (1978), "An Exploratory Study of the Influences of UsageSituation on Perceptions of Product Markets," in Advances in Consumer Research, Vol. 5, ed. H. Keith Hunt, Chicago: Association for Consumer Research, 32-38.

Stefflre, Volney J. (1979), "New Products: Organizational and Technical Problems and Opportunities," in Analytical Approaches to Product and Marketing Planning, ed. Allan D. Shocker, Cambridge, MA: Marketing Science Institute, 415-80.

Tversky, Amos and Daniel Kahneman (1991), "Loss Aversion in Riskless Choice: A Reference-Dependent Model," Quarterly Journal of Economics, 106 (November), 1039-62.

Wedel, Michel and Wagner A. Kamakura (2000), Market Segmentation: Conceptual and Methodological Foundations, Boston: Kluwer.

Wedel, Michel and Cor Kistemaker (1989), "Consumer Benefit Segmentation Using Clusterwise Linear Regression," International Journal of Research in Marketing, 6 (1), 45-59. 
Copyright of Journal of Consumer Research is the property of Journal of Consumer Research. Inc. and its content may not be copied or emailed to multiple sites or posted to a listserv without the copyright holder's express written permission. However, users may print, download, or email articles for individual use. 